\title{
Singularly Perturbed Reaction-Diffusion Problems as First order systems
}

\author{
Sebastian Franz ${ }^{1}$ (D)
}

Received: 15 March 2021 / Revised: 3 August 2021 / Accepted: 5 September 2021 /

Published online: 5 October 2021

(c) The Author(s) 2021

\section{Abstract}

We consider a singularly perturbed reaction diffusion problem as a first order two-by-two system. Using piecewise discontinuous polynomials for the first component and $H_{\mathrm{div}^{-}}$ conforming elements for the second component we provide a convergence analysis on layer adapted meshes and an optimal convergence order in a balanced norm that is comparable with a balanced $H^{2}$-norm for the second order formulation.

Keywords Reaction diffusion problem · Singularly perturbed · First order system ·

Balanced norm

Mathematics Subject Classification $65 \mathrm{~N} 12 \cdot 65 \mathrm{~N} 15 \cdot 65 \mathrm{~N} 30$

\section{Introduction}

Consider the singularly perturbed reaction diffusion problem, given in $\Omega=(0,1)^{2}$ by

$$
-\varepsilon^{2} \Delta u+c u=f,
$$

where $0<\varepsilon \ll 1, c \in W^{1, \infty}, c_{\infty} \geq c \geq c_{0}>0$ and $u=0$ on $\partial \Omega$. We rewrite the problem, using $\boldsymbol{u}=-\varepsilon \operatorname{grad}^{\circ} u$, into a first order system

$$
\left[\left(\begin{array}{ll}
c & 0 \\
0 & 1
\end{array}\right)+\left(\begin{array}{cc}
0 & \varepsilon \operatorname{div} \\
\varepsilon \operatorname{grad}^{\circ} & 0
\end{array}\right)\right]\left(\begin{array}{l}
u \\
\boldsymbol{u}
\end{array}\right)=\left(\begin{array}{l}
f \\
0
\end{array}\right),
$$

where $\operatorname{grad}^{\circ}$ denotes the gradient in $H_{0}^{1}(\Omega)$ and div its adjoint, the divergence. This formulation is also called a mixed formulation. For its weak formulation let $\langle\cdot, \cdot\rangle$ denote the $L^{2}$-scalar product over $\Omega$. Then (2) becomes with $V=(v, v) \in L^{2}(\Omega) \times H_{\text {div }}(\Omega)$

$$
\langle c u, v\rangle+\varepsilon\langle\operatorname{div} \boldsymbol{u}, v\rangle+\langle\boldsymbol{u}, \boldsymbol{v}\rangle+\varepsilon\left\langle\operatorname{grad}^{\circ} u, \boldsymbol{v}\right\rangle=\langle f, v\rangle
$$

Sebastian Franz

sebastian.franz@tu-dresden.de

1 Institute of Scientific Computing, Technische Universität Dresden, Dresden, Germany 
which can also be written for $U=(u, \boldsymbol{u}) \in L^{2}(\Omega) \times H_{\text {div }}(\Omega)$ as

$$
B(U, V):=\langle c u, v\rangle+\varepsilon\langle\operatorname{div} \boldsymbol{u}, v\rangle+\langle\boldsymbol{u}, \boldsymbol{v}\rangle-\varepsilon\langle u, \operatorname{div} \boldsymbol{v}\rangle=\langle f, v\rangle .
$$

This is the weak form we will discretise and analyse.

Singularly perturbed reaction diffusion problems were analysed in many papers, see e.g. $[2,18]$. The associated norm to (1) is the $\varepsilon$-weighted $H^{1}$-norm, also called energy norm. Unfortunately, that norm is not strong enough to see the boundary layers. For example, the corresponding layer function for the boundary $x=0$ is of the type $\mathrm{e}^{-x / \varepsilon}$. Here it holds

$$
\left\|\mathrm{e}^{-x / \varepsilon}\right\|_{L^{2}(\Omega)}+\varepsilon\left\|\operatorname{grad} \mathrm{e}^{-x / \varepsilon}\right\|_{L^{2}(\Omega)} \lesssim \varepsilon^{1 / 2} \stackrel{\varepsilon \rightarrow 0}{\longrightarrow} 0 .
$$

Therefore over the last years convergence in a balanced norm, where the boundary layers do not vanish for $\varepsilon \rightarrow 0$, was considered, see [1,9,11,17]. For the lowest order Raviart-Thomas elements on a Shishkin mesh the system (3) was also considered in [15, Section 5] and analysed in a balanced $H^{1}$-comparable norm.

In this paper we prove optimal convergence orders in a stronger balanced $\mathrm{H}^{2}$-comparable norm

$$
\|\| U\left\|_{b a l}^{2} \sim\right\| u\left\|_{L^{2}(\Omega)}^{2}+\varepsilon^{-1}\right\| \boldsymbol{u}\left\|_{L^{2}(\Omega)}^{2}+\varepsilon\right\| \operatorname{div} \boldsymbol{u} \|_{L^{2}(\Omega)}^{2}
$$

for a variety of $H_{\mathrm{div}}$-conforming elements on general layer-adapted meshes. The paper is organised as follows. In Sect. 2 we define the numerical method and recall results for a solution decomposition and interpolation errors. In Sect. 3 we provide the convergence analysis and in the final Sect. 4 some numerical examples illustrating our theoretical results are given.

\subsection{Notation}

We denote vector valued functions with a bold font. $L^{p}(D)$ with the norm $\|\cdot\|_{L^{p}(D)}$ is the classical Lebesgue space of function integrable to the power $p$ over a domain $D \subset \mathbb{R}^{2}$ and $W^{\ell, p}(D)$ the corresponding Sobolev space for derivatives up to order $\ell$. Furthermore, we write $A \lesssim B$ if there exists a generic constant $C>0$ such that $A \leq C \cdot B$.

\section{Numerical Method and Interpolation Errors}

In order to define our numerical method, we need discrete spaces defined over an appropriate mesh. A basic tool for defining this mesh is the knowledge of a solution decomposition, especially the structure of layers.

Assumption 1 The solution $u$ of (1) can be written as

$$
u=s+w_{1}+w_{2}+w_{3}+w_{4}+w_{12}+w_{23}+w_{34}+w_{41}
$$

where $s$ is the smooth part, $w_{i}$ are boundary layers and $w_{i j}$ are corner layers (both counted counterclockwise). To be more precise, for any given degree $k$ it holds for $0 \leq i, j \leq k+2$,

$$
\left\|\partial_{x}^{i} \partial_{y}^{j} s\right\|_{L^{\infty}(\Omega)} \lesssim 1, \quad\left|\partial_{x}^{i} \partial_{y}^{j} w_{1}(x, y)\right| \lesssim \varepsilon^{-i} \mathrm{e}^{-x / \varepsilon}, \quad\left|\partial_{x}^{i} \partial_{y}^{j} w_{12}(x, y)\right| \lesssim \varepsilon^{-(i+j)} \mathrm{e}^{-(x+y) / \varepsilon},
$$

and analogously for the other boundary layers and corner layers.

Remark 2 Using above solution decomposition for $u$ we derive a similar decomposition for the solution $U$ of (3), as $U=(u, \boldsymbol{u})$ and $\boldsymbol{u}=-\varepsilon \operatorname{grad} u$. 
Such assumptions on a solution decomposition are very common in the analysis of singularly perturbed problems. They hold true under compatibility and regularity conditions on the data, see e.g. $[6,10,13]$.

We follow [16] and construct an S-type mesh using the information of the solution decomposition. First, we define a transition point $\lambda$, such that a typical boundary layer function is small enough:

$$
\exp (-\lambda / \varepsilon)=N^{-\sigma} \Rightarrow \lambda=\sigma \varepsilon \ln (N),
$$

for a constant $\sigma>0$ specified later. We additionally assume

$$
\lambda=\min \left\{\sigma \varepsilon \ln (N), \frac{1}{4}\right\},
$$

as otherwise $\varepsilon$ is large enough to facilitate a standard numerical analysis. Now $0=x_{0}<$ $x_{1}<\cdots<x_{N}=1$ are given by

$$
x_{i}:= \begin{cases}\sigma \varepsilon \phi\left(\frac{2 i}{N}\right), & i=0, \ldots, N / 4, \\ \frac{2 i}{N}(1-2 \lambda)-\frac{1}{2}+2 \lambda, & i=N / 4, \ldots, 3 N / 4, \\ 1-\sigma \varepsilon \phi\left(2-\frac{2 i}{N}\right), & i=3 N / 4, \ldots, N,\end{cases}
$$

where $\phi$ is a mesh-generating function with the properties

$-\phi$ is monotonically increasing,

$-\phi(0)=0$ and $\phi(1 / 2)=\ln N$,

- $\phi$ is piecewise differentiable with $\max \phi^{\prime} \leq C N$ and

$-\min _{i=1, \ldots, N / 4}\left(\phi\left(\frac{2 i}{N}\right)-\phi\left(\frac{2(i-1)}{N}\right)\right) \geq C N^{-1}$.

The first three conditions are given in [16], while the last one allows the mesh-widths inside the boundary layers to be bounded from below, see also [8]. Related to $\phi$ we define the mesh characterising function $\psi$ by

$$
\psi=\mathrm{e}^{-\phi} .
$$

Several S-type meshes are given in [16] fulfilling above properties. We only provide the definitions of the two mostly used. For the Shishkin mesh we have

$$
\phi(t)=2 t \ln N, \quad \psi(t)=N^{-2 t}, \quad \max \left|\psi^{\prime}\right|=2 \ln N
$$

and the Bakhvalov-S-mesh

$$
\phi(t)=-\ln \left(1-2 t\left(1-N^{-1}\right)\right), \quad \psi(t)=1-2 t\left(1-N^{-1}\right), \quad \max \left|\psi^{\prime}\right|=2 .
$$

In addition to the mesh generating and characterising functions also $\max \left|\psi^{\prime}\right|:=$ $\max _{t \in[0,1 / 2]}\left|\psi^{\prime}(t)\right|$ is given, that enters all the error estimates on S-type meshes.

The two-dimensional mesh $T_{N}$ is then defined by all cells $K_{i j}:=\left(x_{i-1}, x_{i}\right) \times\left(x_{j-1}, x_{j}\right)$ for $1 \leq i, j \leq N$. Note that it holds

$$
h_{i}:=x_{i}-x_{i-1} \lesssim \begin{cases}\varepsilon N^{-1} \max \left|\psi^{\prime}\right| e^{x /(\sigma \varepsilon)}, & i \leq N / 4 \text { or } i>3 N / 4, \text { and } x \in\left[x_{i-1}, x_{i}\right], \\ N^{-1}, & \text { otherwise, }\end{cases}
$$


where the first estimate can e.g. be found in [12, Lemma 2.3]. It also follows the simpler bound

$$
h:=\max _{i=1, \ldots, N / 4} h_{i} \lesssim \varepsilon .
$$

Let us denote two subdomains of $\Omega$ per layer function, exemplarily given for $w_{1}$ by

$$
\Omega_{1}:=[0, \lambda] \times[0,1] \text { and } \Omega_{1}^{*}:=\left[0, x_{N / 4-1}\right] \times[0,1] \subset \Omega_{1}
$$

and for $w_{12}$ by

$$
\Omega_{12}:=[0, \lambda]^{2} \quad \text { and } \quad \Omega_{12}^{*}:=\left[0, x_{N / 4-1}\right]^{2} \subset \Omega_{12} .
$$

With (3) only needing $L^{2}$-regularity for the first component and $H_{\text {div }}$-regularity for the second component, our discrete spaces are

$$
\mathcal{U}_{N}:=\left\{\left(u_{N}, \boldsymbol{u}_{N}\right) \subset L^{2}(\Omega) \times H_{\operatorname{div}}(\Omega): \forall K \subset T_{N}:\left.u_{N}\right|_{K} \in \mathcal{Q}_{k}(K),\left.\boldsymbol{u}_{N}\right|_{K} \in \mathcal{D}_{k}(K)\right\},
$$

where $\mathcal{Q}_{k}(K)$ is the space of polynomials with degree up to $k$ in each variable on the cell $K$ of $T_{N}$. For the discretisation of $H_{\text {div }}$ with $\mathcal{D}_{k}(K)$ we can use

- the Raviart-Thomas space

$$
R T_{k}(K)=\mathcal{Q}_{k+1, k}(K) \times \mathcal{Q}_{k, k+1}(K),
$$

introduced by Raviart and Thomas in [14] on triangular meshes, see also [4] for rectangular meshes, where $\mathcal{Q}_{p, q}(K)$ is the space of polynomials with degree $p$ in $x$ and degree $q$ in $y$ on the cell $K$ or

- the Brezzi-Douglas-Marini space

$$
B D M_{k}(K):=\left(\mathcal{P}_{k}(K)\right)^{2} \oplus \operatorname{span}\left\{\operatorname{curl}\left(x^{k+1} y\right), \operatorname{curl}\left(x y^{k+1}\right)\right\},
$$

see [5], where $\mathcal{P}_{k}(K)$ is the space of polynomials of total degree $k$ on the cell $K$ and $\operatorname{curl} w=\left(\partial_{y} w,-\partial_{x} w\right)$.

Then the discrete method reads: Find $U_{N}=\left(u_{N}, \boldsymbol{u}_{N}\right) \in \mathcal{U}_{N}$, s.t. for all $V \in \mathcal{U}_{N}$ it holds

$$
B\left(U_{N}, V\right)=\left\langle c u_{N}, v\right\rangle+\varepsilon\left\langle\operatorname{div} \boldsymbol{u}_{N}, v\right\rangle+\left\langle\boldsymbol{u}_{N}, \boldsymbol{v}\right\rangle-\varepsilon\left\langle u_{N}, \operatorname{div} \boldsymbol{v}\right\rangle=\langle f, v\rangle .
$$

Note that the solution $U$ of (3) does also fulfill (5) and we therefore have Galerkin orthogonality

$$
B\left(U_{N}-U, V\right)=0, \quad \forall V \in \mathcal{U}_{N}
$$

\section{Numerical Analysis}

Let us start with an interpolation operator into $\mathcal{U}_{N}$ given by its two components. The first one $\mathcal{I}_{1}$ will be a weighted local $L^{2}$-projection, defined on any $K \subset T_{N}$ by

$$
\left\langle c\left(\mathcal{I}_{1} u-u\right), w\right\rangle_{K}=0 \text { for all } w \in \mathcal{Q}_{k}(K) .
$$

This weighted $L^{2}$-projection is $L^{2}$-stable due to $0<c_{0} \leq c \leq c_{\infty}$ and

$$
\begin{aligned}
& \left\|c^{1 / 2} \mathcal{I}_{1} v\right\|_{L^{2}(K)}^{2}=\left\langle c \mathcal{I}_{1} v, \mathcal{I}_{1} v\right\rangle_{K}=\left\langle c v, \mathcal{I}_{1}, v\right\rangle_{K} \leq\left\|c^{1 / 2} v\right\|_{L^{2}(K)}\left\|c^{1 / 2} \mathcal{I}_{1} v\right\|_{L^{2}(K)} \\
& \Rightarrow\left\|\mathcal{I}_{1} v\right\|_{L^{2}(K)} \leq \frac{1}{c_{0}^{1 / 2}}\left\|c^{1 / 2} \mathcal{I}_{1} v\right\|_{L^{2}(K)} \leq \frac{1}{c_{0}^{1 / 2}}\left\|c^{1 / 2} v\right\|_{L^{2}(K)} \leq\left(\frac{c_{\infty}}{c_{0}}\right)^{1 / 2}\|v\|_{L^{2}(K)}
\end{aligned}
$$


for all $v \in L^{2}(K)$. Moreover, following [17] and denoting by $\mathcal{L}_{1}$ a pointwise Lagrange interpolation operator into $\mathcal{Q}_{k}(K)$ we obtain

$$
\begin{aligned}
\left\|\mathcal{I}_{1} v-v\right\|_{L^{2}(K)} & \leq\left\|\mathcal{I}_{1} v-\mathcal{I}_{1} \mathcal{L}_{1} v\right\|_{L^{2}(K)}+\left\|\mathcal{I}_{1} \mathcal{L}_{1} v-v\right\|_{L^{2}(K)} \\
& =\left\|\mathcal{I}_{1}\left(v-\mathcal{L}_{1} v\right)\right\|_{L^{2}(K)}+\left\|\mathcal{L}_{1} v-v\right\|_{L^{2}(K)} \\
& \leq\left(\left(\frac{c_{\infty}}{c_{0}}\right)^{1 / 2}+1\right)\left\|\mathcal{L}_{1} v-v\right\|_{L^{2}(K)} .
\end{aligned}
$$

By using the interpolation error results of [2] for the remaining error, the anisotropic interpolation error estimates follow, i.e. for any $0 \leq \ell \leq k+1$ it holds on a cell $K$ with dimension $h_{x} \times h_{y}$

$$
\left\|\mathcal{I}_{1} v-v\right\|_{L^{2}(K)} \lesssim h_{x}^{\ell}\left\|\partial_{x}^{\ell} v\right\|_{L^{2}(K)}+h_{y}^{\ell}\left\|\partial_{y}^{\ell} v\right\|_{L^{2}(K)}
$$

if $v \in H^{\ell}(K)$.

The second operator $\mathcal{I}_{2}$ utilises the classical interpolation operator $\mathcal{J}$ on $\mathcal{D}_{k}$. It is defined on each cell $K$ for

$-\mathcal{D}_{k}(K)=R T_{k}(K)$ by

$$
\begin{gathered}
\int_{F}(\mathcal{J} \boldsymbol{v}-\boldsymbol{v}) \cdot \boldsymbol{n} \cdot q=0, \quad \forall q \in \mathcal{P}_{k}(F) \text { for all faces } F \subset \partial K, \\
\int_{K}(\mathcal{J} \boldsymbol{v}-\boldsymbol{v}) \cdot \boldsymbol{q}=0, \quad \forall \boldsymbol{q} \in \mathcal{Q}_{k-1, k}(K) \times \mathcal{Q}_{k, k-1}(K),
\end{gathered}
$$

- $\mathcal{D}_{k}(K)=B D M_{k}(K)$ by

$$
\begin{gathered}
\int_{F}(\mathcal{J} \boldsymbol{v}-\boldsymbol{v}) \cdot \boldsymbol{n} \cdot q=0, \quad \forall q \in \mathcal{P}_{k}(F), \forall F \subset \partial K, \\
\int_{K}(\mathcal{J} \boldsymbol{v}-\boldsymbol{v}) \cdot \boldsymbol{q}=0, \quad \forall \boldsymbol{q} \in\left(\mathcal{P}_{k-2}(K)\right)^{2} .
\end{gathered}
$$

It holds the anisotropic interpolation error estimates for $\mathcal{J}$, see [7,19],

$$
\|\mathcal{J} \boldsymbol{v}-\boldsymbol{v}\|_{L^{2}(K)} \lesssim \sum_{s=0}^{k+1} h_{x}^{k+1-s} h_{y}^{s}\left\|\partial_{x}^{k+1-s} \partial_{y}^{s} \boldsymbol{v}\right\|_{L^{2}(K)}
$$

if $v \in H^{k+1}(K)$. Note that for $R T_{k}$ an even sharper result involving only pure derivatives of $v$ holds, see [7]. In addition, we have also anisotropic interpolation error estimates for the $L^{2}$-norm of the divergence, see [7],

$$
\begin{aligned}
R T_{k}: \quad\|\operatorname{div}(\mathcal{J} \boldsymbol{v}-\boldsymbol{v})\|_{L^{2}(K)} & \lesssim h_{x}^{k+1}\left\|\partial_{x}^{k+1} \operatorname{div} \boldsymbol{v}\right\|_{L^{2}(K)}+h_{y}^{k+1}\left\|\partial_{y}^{k+1} \operatorname{div} \boldsymbol{v}\right\|_{L^{2}(K)}, \\
B D M_{k}: \quad\|\operatorname{div}(\mathcal{J} \boldsymbol{v}-\boldsymbol{v})\|_{L^{2}(K)} & \lesssim \sum_{|\boldsymbol{\alpha}|=k} h_{x}^{\alpha_{1}} h_{y}^{\alpha_{2}}\left\|\partial_{x}^{\alpha_{1}} \partial_{y}^{\alpha_{2}} \operatorname{div} \boldsymbol{v}\right\|_{L^{2}(K)},
\end{aligned}
$$

if $\boldsymbol{v}$ is such that $\operatorname{div} v \in H^{k+1}(K)$ for $R T_{k}$ and $\operatorname{div} v \in H^{k}(K)$ for $B D M_{k}$.

If we only want to prove convergence in the $L^{2}$-norm of $U=(u,-\varepsilon \operatorname{grad} u)$ the interpolation operator $\mathcal{J}$ is enough. For a stronger convergence result we define a more 
sophisticated operator, following ideas from [11]. Recalling the decomposition of $u$, we have for $\boldsymbol{u}=-\varepsilon \operatorname{grad} u$ the decomposition

$$
u=s+w^{1}+w^{2}+w^{3}+w^{4}+w^{12}+w^{23}+w^{34}+w^{41}
$$

with the obvious definition of the bold font letters. Now the operator $\mathcal{I}_{2}$ is defined piecewise on each cell $K \subset T_{N}$ with $i d \in\{1,2,3,4,12,23,34,41\}$

$$
\left.\mathcal{I}_{2} \boldsymbol{s}\right|_{K}:=\left.\mathcal{J} \boldsymbol{s}\right|_{K},\left.\quad \mathcal{I}_{2} \boldsymbol{w}^{i d}\right|_{K}:= \begin{cases}\left.\mathcal{J} \boldsymbol{w}^{i d}\right|_{K}, & K \subset \Omega_{i d}^{*}, \\ \left.\hat{\mathcal{J}}^{i d} \boldsymbol{w}^{i d}\right|_{K}, & K \subset \Omega_{i d} \backslash \Omega_{i d}^{*}, \\ 0, & K \subset \Omega \backslash \Omega_{i d} .\end{cases}
$$

Using $\Gamma_{i d}:=\partial \Omega_{i d} \backslash \Gamma$ we define the remaining operators on each $K \subset \Omega_{i d} \backslash \Omega_{i d}^{*}$ using the same definition as for $\mathcal{J}$ with the exception of the first condition in (8) and (9). This one is replaced by the two conditions

$$
\begin{aligned}
\int_{F}\left(\hat{\mathcal{J}}^{i d} \boldsymbol{w}^{i d}-\boldsymbol{w}^{i d}\right) \cdot \boldsymbol{n} \cdot q & =0 \text { for each face } F \subset \partial K \backslash \Gamma_{i d}, \forall q \in \mathcal{P}_{k}(F), \\
\int_{F} \hat{\mathcal{J}}^{i d} \boldsymbol{w}^{i d} \cdot \boldsymbol{n} \cdot q & =0 \text { for each face } F \subset \partial K \cap \Gamma_{i d}, \forall q \in \mathcal{P}_{k}(F) .
\end{aligned}
$$

For our analysis let us define a norm that is associated with $B(\cdot, \cdot)$. Here it holds

$$
B(U, U) \geq \min \left\{1, c_{0}\right\}\|U\|_{L^{2}(\Omega)}^{2}
$$

that is equivalent to coercivity in the energy norm of the weak formulation of (1). But we can actually use the stronger norm

$$
\|U \mid\|:=\left(\|U\|_{L^{2}(\Omega)}^{2}+\delta\|\varepsilon \operatorname{div} \boldsymbol{u}\|_{L^{2}(\Omega)}^{2}\right)^{1 / 2},
$$

where $\delta \leq \frac{c_{0}}{c_{\infty}^{2}}$. This norm is equivalent to the weighted $H^{2}$-norm $\|u\|_{L^{2}(\Omega)}+$ $\varepsilon\|\operatorname{grad} u\|_{L^{2}(\Omega)}+\varepsilon^{2}\|\Delta u\|_{L^{2}(\Omega)}$, which is stronger than the energy norm and, unfortunately, also not balanced. To repair this weakness we also introduce a balanced version of this norm

$$
\|\| U \|_{\text {bal }}:=\left(\|u\|_{L^{2}(\Omega)}^{2}+\varepsilon^{-1}\|\boldsymbol{u}\|_{L^{2}(\Omega)}^{2}+\delta \varepsilon^{-1}\|\varepsilon \operatorname{div} \boldsymbol{u}\|_{L^{2}(\Omega)}^{2}\right)^{1 / 2},
$$

that is also considered in [11]. The remainder of this section is devoted to proving optimal uniform convergence orders in the balanced norm. Of course, convergence in the unbalanced norm then follows.

Lemma 3 For each $\delta \leq \frac{c_{0}}{c_{\infty}^{2}}$ exists a constant $\beta>0$, such that for all $V \in \mathcal{U}_{N}$ it holds

$$
\sup _{\chi \in \mathcal{U}_{N}} \frac{B(V, \chi)}{\|\chi\|_{L^{2}(\Omega)}} \geq \beta\|\| V \| \mid .
$$

Proof By (13) we already have

$$
B(V, V) \geq \min \left\{1, c_{0}\right\}\|V\|_{L^{2}(\Omega)}^{2} .
$$

Choosing as test function $\chi(V)=(v+\delta \varepsilon \operatorname{div} \boldsymbol{v}, \boldsymbol{v}) \in \mathcal{U}_{N}$, we obtain

$$
B(V, \chi(V)) \geq\left(c_{0}-\delta \frac{c_{\infty}^{2}}{2}\right)\|v\|_{L^{2}(\Omega)}^{2}+\|\boldsymbol{v}\|_{L^{2}(\Omega)}^{2}+\frac{\delta}{2}\|\varepsilon \operatorname{div} \boldsymbol{v}\|_{L^{2}(\Omega)}^{2}
$$


and together with $\delta \leq \frac{c_{0}}{c_{\infty}^{2}}$ we have

$$
B(V, \chi(V)) \geq \frac{c_{0}}{2}\|v\|_{L^{2}(\Omega)}^{2}+\|\boldsymbol{v}\|_{L^{2}(\Omega)}^{2}+\frac{\delta}{2}\|\varepsilon \operatorname{div} \boldsymbol{v}\|_{L^{2}(\Omega)}^{2} \geq \frac{1}{2} \min \left\{c_{0}, 2\right\}\|\| V\|\|^{2} .
$$

In addition it holds

$$
\|\chi(V)\|_{L^{2}(\Omega)}^{2} \leq 2\|V\|_{L^{2}(\Omega)}^{2}+2 \delta^{2}\|\varepsilon \operatorname{div} \boldsymbol{v}\|_{L^{2}(\Omega)}^{2} \leq 2 \max \{1, \delta\}\|V\| \|^{2} .
$$

Thus it follows

$$
\sup _{\chi \in \mathcal{U}_{N}} \frac{B(V, \chi)}{\|\chi\|_{L^{2}(\Omega)}} \geq \frac{B(V, \chi(V))}{\|\chi(V)\|_{L^{2}(\Omega)}} \geq \frac{\sqrt{2}}{4} \frac{\min \left\{2, c_{0}\right\}}{\max \{1, \sqrt{\delta}\}}\|V\| \| .
$$

Setting $\beta=\frac{\sqrt{2}}{4} \frac{\min \left\{2, c_{0}\right\}}{\max \{1, \sqrt{\delta}\}} \geq \frac{\sqrt{2}}{4} \frac{\min \left\{2, c_{0}\right\}}{\max \left\{1, \frac{\sqrt{c_{0}}}{c_{\infty}}\right\}}>0$ proves the assertion.

Let us split the error $U-U_{N}$ into an interpolation error and a discrete error

$$
U-U_{N}=U-\mathcal{I} U+\mathcal{I} U-U_{N}=:(\eta, \eta)-(\xi, \xi),(\xi, \xi) \in \mathcal{U}_{N} .
$$

Using above inf-sup inequality and the Galerkin orthogonality (6) we arrive at

$$
\beta|\|(\xi, \xi)\|| \leq \sup _{V \in \mathcal{U}_{N}} \frac{B((\xi, \xi), V)}{\|V\|_{L^{2}(\Omega)}}=\sup _{V \in \mathcal{U}_{N}} \frac{B((\eta, \boldsymbol{\eta}), V)}{\|V\|_{L^{2}(\Omega)}},
$$

and we are left with estimating $B((\eta, \eta), V)$ for any $V \in \mathcal{U}_{N}$. Here it holds using (5)

$$
\begin{aligned}
B((\eta, \eta), V) & =\langle c \eta, v\rangle+\varepsilon\langle\operatorname{div} \boldsymbol{\eta}, v\rangle+\langle\boldsymbol{\eta}, \boldsymbol{v}\rangle-\varepsilon\langle\eta, \operatorname{div} \boldsymbol{v}\rangle \\
& =\varepsilon\langle\operatorname{div} \boldsymbol{\eta}, v\rangle+\langle\boldsymbol{\eta}, \boldsymbol{v}\rangle-\varepsilon\langle\eta, \operatorname{div} \boldsymbol{v}\rangle
\end{aligned}
$$

due to $\mathcal{I}_{1}$ being the weighted $L^{2}$-projection. Note that in the case of constant $c$, the last term would also vanish due to $\left.\operatorname{div} \boldsymbol{v}\right|_{K} \in \mathcal{Q}_{k}(K)$.

Lemma 4 It holds for $\sigma>k+1$

$$
\|\boldsymbol{\eta}\|_{L^{2}(\Omega)} \lesssim \varepsilon^{1 / 2}\left(h+N^{-1} \max \left|\psi^{\prime}\right|\right)^{k+1} .
$$

In the case of $\mathcal{D}(K)=R T_{k}(K)$ and $\sigma \geq k+3 / 2$ we obtain

$$
\|\operatorname{div} \eta\|_{L^{2}(\Omega)} \lesssim \varepsilon^{-1 / 2}\left(h+N^{-1} \max \left|\psi^{\prime}\right|\right)^{k+1},
$$

while for $\mathcal{D}(K)=B D M_{k}$ and $\sigma \geq k+1 / 2$ we have

$$
\|\operatorname{div} \eta\|_{L^{2}(\Omega)} \lesssim \varepsilon^{-1 / 2}\left(h+N^{-1} \max \left|\psi^{\prime}\right|\right)^{k} .
$$

Proof Using the solution decomposition (12) and the anisotropic interpolation error estimate (10) we obtain

$$
\left\|\mathcal{I}_{2} \boldsymbol{s}-\boldsymbol{s}\right\|_{L^{2}(\Omega)}=\|\mathcal{J} \boldsymbol{s}-\boldsymbol{s}\|_{L^{2}(\Omega)} \lesssim\left(h+N^{-1}\right)^{k+1}\|\boldsymbol{s}\|_{H^{k+1}(\Omega)} \lesssim \varepsilon\left(h+N^{-1}\right)^{k+1} .
$$

For the boundary layer terms we use the special structure of $\mathcal{I}_{2}$ and estimate differently on the subdomains of $\Omega$. We show the procedure for $\boldsymbol{w}^{1}$, the estimates of the other terms follow similarly. In $\Omega \backslash \Omega_{1}$ the interpolant is zero and we get

$$
\left\|\mathcal{I}_{2} \boldsymbol{w}^{1}-\boldsymbol{w}^{1}\right\|_{L^{2}\left(\Omega \backslash \Omega_{1}\right)}=\left\|\boldsymbol{w}^{1}\right\|_{L^{2}\left(\Omega \backslash \Omega_{1}\right)} \lesssim \varepsilon^{1 / 2} N^{-\sigma} .
$$

In $\Omega_{1}$ we have

$$
\left\|\mathcal{I}_{2} \boldsymbol{w}^{1}-\boldsymbol{w}^{1}\right\|_{L^{2}\left(\Omega_{1}\right)} \leq\left\|\mathcal{J} \boldsymbol{w}^{1}-\boldsymbol{w}^{1}\right\|_{L^{2}\left(\Omega_{1}\right)}+\left\|\mathcal{P}^{1} \boldsymbol{w}^{1}\right\|_{L^{2}\left(\Omega_{1} \backslash \Omega_{1}^{*}\right)},
$$


where $\mathcal{P}^{1}:=\hat{\mathcal{J}}^{1}-\mathcal{J}$. For the first term we obtain using (10) and (4)

$$
\begin{aligned}
\left\|\mathcal{J} \boldsymbol{w}^{1}-\boldsymbol{w}^{1}\right\|_{L^{2}\left(\Omega_{1}\right)}^{2} & \lesssim \sum_{K \subset \Omega_{1}} \sum_{\ell=0}^{k+1} \varepsilon^{2(k+1-\ell)}\left(N^{-1} \max \left|\psi^{\prime}\right|\right)^{2(k+1-\ell)} N^{-2 \ell} \times \\
& \lesssim\left(\mathrm{e}^{\frac{(k+1-\ell) x}{\sigma \varepsilon}} \varepsilon^{-1} \max \left|\psi^{\prime}\right|\right)^{2(k+1)}\left\|\mathrm{e}^{\frac{(k+1-\sigma) x}{\sigma \varepsilon}}\right\|_{L^{2}\left(\Omega_{1}\right)}^{2} \mathrm{e}^{-\frac{x}{\varepsilon}} \|_{L^{2}(K)}^{2} \\
& \lesssim\left(N^{-1} \max \left|\psi^{\prime}\right|\right)^{2(k+1)} \varepsilon,
\end{aligned}
$$

due to $\sigma>k+1$. In the remaining ply of elements the operator $\mathcal{P}^{1}$ in the Raviart-Thomas case is given by

$$
\begin{aligned}
& \int_{F} \mathcal{P}^{1} \boldsymbol{w}^{1} \cdot \boldsymbol{n} \cdot q=0 \text { for all faces } F \subset \partial K \backslash \Gamma_{1}, \forall q \in \mathcal{P}_{k}(F), \\
& \int_{F} \mathcal{P}^{1} \boldsymbol{w}^{1} \cdot \boldsymbol{n} \cdot q=\int_{F} \boldsymbol{w}^{1} \cdot \boldsymbol{n} \cdot q \text { for all faces } F \subset \partial K \cap \Gamma_{1}, \forall q \in \mathcal{P}_{k}(F), \\
& \int_{K}\left(\mathcal{P}^{1} \boldsymbol{w}^{1}\right) \cdot \boldsymbol{q}=0, \forall \boldsymbol{q} \in \mathcal{Q}_{k-1, k}(K) \times \mathcal{Q}_{k, k-1}(K),
\end{aligned}
$$

and similarly for the other finite elements. Thus $\mathcal{P}^{1} \boldsymbol{w}^{1}$ depends only on $\left.\boldsymbol{w}^{1} \cdot \boldsymbol{n}\right|_{\Gamma_{1}}$. With $\mathcal{P}^{1}$ on $\Gamma_{1}$ being defined by weighted integrals, we have

$$
\left\|\mathcal{P}^{1} \boldsymbol{w}^{1}\right\|_{L^{2}\left(\Omega_{1} \backslash \Omega_{1}^{*}\right)} \lesssim \operatorname{meas}\left(\Omega_{1} \backslash \Omega_{1}^{*}\right)^{1 / 2}\left\|\boldsymbol{w}^{1} \cdot \boldsymbol{n}\right\|_{L^{\infty}\left(\Gamma_{1}\right)} \lesssim h_{N / 4}^{1 / 2} N^{-\sigma} \lesssim \varepsilon^{1 / 2} N^{-(k+1)} .
$$

Applying the same techniques to the other boundary and corner layer terms, and collecting the result finishes the first part of the proof.

For the divergence we can apply the same techniques with the difference of applying (11) instead of (10). We obtain for $\sigma>k+1$ and $\mathcal{D}(K)=R T_{k}(K)$

$$
\begin{aligned}
\left\|\operatorname{div}\left(\mathcal{I}_{2} \boldsymbol{s}-\boldsymbol{s}\right)\right\|_{L^{2}(\Omega)} & \lesssim\left(h+N^{-1}\right)^{k+1}\|\operatorname{div} \boldsymbol{s}\|_{H^{k+1}(\Omega)} \lesssim \varepsilon\left(h+N^{-1}\right)^{k+1}, \\
\left\|\operatorname{div}\left(\mathcal{I}_{2} \boldsymbol{w}^{1}-\boldsymbol{w}^{1}\right)\right\|_{L^{2}\left(\Omega \backslash \Omega_{1}\right)} & =\left\|\operatorname{div} \boldsymbol{w}^{1}\right\|_{L^{2}\left(\Omega \backslash \Omega_{1}\right)} \lesssim \varepsilon^{-1 / 2} N^{-\sigma}, \\
\left\|\operatorname{div}\left(\mathcal{J} \boldsymbol{w}^{1}-\boldsymbol{w}^{1}\right)\right\|_{L^{2}\left(\Omega_{1}\right)} & \lesssim \varepsilon^{-1}\left(N^{-1} \max \left|\psi^{\prime}\right|\right)^{k+1}\left\|\mathrm{e}^{\frac{(k+1-\sigma) x}{\sigma \varepsilon}}\right\|_{L^{2}\left(\Omega_{1}\right)} \\
& \lesssim \varepsilon^{-1 / 2}\left(N^{-1} \max \left|\psi^{\prime}\right|\right)^{k+1} .
\end{aligned}
$$

The last term to estimate is the error on the ply of elements in $\Omega_{1} \backslash \Omega_{1}^{*}$. A closer inspection of $\mathcal{P}^{1} \boldsymbol{w}^{1}$ reveals $\left(\mathcal{P}^{1} \boldsymbol{w}^{1}\right)_{2}=0$. Thus, an inverse inequality followed by (15) yields

$$
\begin{aligned}
\left\|\operatorname{div}\left(\mathcal{P}^{1} \boldsymbol{w}^{1}\right)\right\|_{L^{2}\left(\Omega_{1} \backslash \Omega_{1}^{*}\right)} & \lesssim h_{N / 4}^{-1}\left\|\left(\mathcal{P}^{1} \boldsymbol{w}^{1}\right)_{1}\right\|_{L^{2}\left(\Omega_{1} \backslash \Omega_{1}^{*}\right)} \lesssim h_{N / 4}^{-1 / 2}\left\|\left(\boldsymbol{w}^{1}\right)_{1}\right\|_{L^{\infty}\left(\Gamma_{1}\right)} \\
& \lesssim \varepsilon^{-1 / 2} N^{1 / 2} N^{-\sigma}
\end{aligned}
$$

where $h_{N / 4} \geq h_{\text {min }} \geq \varepsilon N^{-1}$ holds due to the assumptions on $\phi$. The analysis for the other terms of the decomposition follows the same lines.

For $\mathcal{D}(K)=B D M_{k}(K)$ the same analysis can be done, only replacing the convergence orders by $k$ for $\sigma \geq k+1 / 2$.

Lemma 5 Assuming $h \varepsilon \lesssim N^{-2}$ and $\sigma \geq k+1$, it holds for any $V=(v, v) \in \mathcal{U}_{N}$

$$
|\langle\eta, \operatorname{div} \boldsymbol{v}\rangle| \lesssim \varepsilon^{-1 / 2}\left(h+N^{-1} \max \left|\psi^{\prime}\right|\right)^{k+1}(\ln N)^{1 / 2}\|\boldsymbol{v}\|_{L^{2}(\Omega)} .
$$


Proof Let $c_{K}:=\frac{1}{\operatorname{meas}(K)} \int_{K} c \geq c_{0}$ be a piecewise constant approximation of $c$. Now

$$
\langle\eta, \operatorname{div} v\rangle=\sum_{K \in T_{N}}\langle\eta, \operatorname{div} \boldsymbol{v}\rangle_{K}=\sum_{K \in T_{N}} \frac{1}{c_{K}}\left\langle\left(c_{K}-c\right) \eta, \operatorname{div} \boldsymbol{v}\right\rangle_{K},
$$

due to the weighted $L^{2}$-projection and $\left.\operatorname{div} \boldsymbol{v}\right|_{K} \in \mathcal{Q}_{k}(K)$. It holds

$$
\left\|c_{K}-c\right\|_{L^{\infty}(K)} \lesssim\left(h_{x}+h_{y}\right)\|c\|_{W^{1, \infty}(K)} .
$$

Thus we obtain, using $h_{x}$ and $h_{y}$ as abbreviations for the dimensions of $K$, an inverse inequality and (7) for any $v \in H^{k+1}(K)$ and $v \in \mathcal{D}_{k}(K)$

$$
\begin{aligned}
& \left|\left\langle\left(c_{K}-c\right)\left(v-\mathcal{I}_{1} v\right), \partial_{x} \boldsymbol{v}_{1}\right\rangle_{K}\right| \\
& \quad \lesssim\left(1+\frac{h_{y}}{h_{x}}\right)\left\|v-\mathcal{I}_{1} v\right\|_{L^{2}(K)}\|\boldsymbol{v}\|_{L^{2}(K)} \\
& \quad \lesssim\left(\left(h_{x}+h_{y}\right)\left\|h_{x}^{k} \partial_{x}^{k+1} v\right\|_{L^{2}(K)}+\left(1+\frac{h_{y}}{h_{x}}\right)\left\|h_{y}^{k+1} \partial_{y}^{k+1} v\right\|_{L^{2}(K)}\right)\|\boldsymbol{v}\|_{L^{2}(K)}
\end{aligned}
$$

and similarly for the $y$-derivative of the second component.

Let us start with the smooth part $s$ of the solution decomposition and denote the coarse part of $\Omega$ by $\Omega_{c}:=\Omega \backslash \bigcup_{i=1}^{4} \Omega_{i}$ and the union of corners by $\Omega_{\text {cor }}:=\Omega_{12} \cup \Omega_{23} \cup \Omega_{34} \cup \Omega_{41}$. Then we obtain by using (16)

$$
\begin{aligned}
\left|\left\langle\left(c_{K}-c\right)\left(s-\mathcal{I}_{1} s\right), \partial_{x} \boldsymbol{v}_{1}\right\rangle\right| \lesssim & \left(h+N^{-1}\right)^{k+1}\left\|\partial_{x}^{k+1} s\right\|_{L^{2}(\Omega)}+N^{-(k+1)}\left\|\partial_{y}^{k+1} s\right\|_{L^{2}\left(\Omega_{c}\right)} \\
& +\varepsilon^{-1} N^{-(k+1)}\left\|\partial_{y}^{k+1} s\right\|_{L^{2}\left(\left(\Omega_{1} \cup \Omega_{3}\right) \backslash \Omega_{c o r}\right)} \\
& +h N h^{k+1}\left\|\partial_{y}^{k+1} s\right\|_{L^{2}\left(\left(\Omega_{2} \cup \Omega_{4}\right) \backslash \Omega_{c o r}\right)} \\
& \left.+h \varepsilon^{-1} N h^{k+1}\left\|\partial_{y}^{k+1} s\right\|_{L^{2}\left(\Omega_{c o r}\right)}\right)\|\boldsymbol{v}\|_{L^{2}(\Omega)} \\
\lesssim & \varepsilon^{-1 / 2}\left(h+N^{-1}\right)^{k+1}\left((\ln N)^{1 / 2}+h \varepsilon^{1 / 2} N \ln N\right)\|\boldsymbol{v}\|_{L^{2}(\Omega)} \\
\lesssim & \varepsilon^{-1 / 2}\left(h+N^{-1}\right)^{k+1}(\ln N)^{1 / 2}\|\boldsymbol{v}\|_{L^{2}(\Omega)}
\end{aligned}
$$

due to $h_{\min } \geq \varepsilon N^{-1}$ and the condition on $h \varepsilon$ implying

$$
h \varepsilon^{1 / 2} \lesssim(h \varepsilon)^{3 / 4} \lesssim N^{-3 / 2}
$$

For $\partial_{y} \boldsymbol{v}_{2}$ holds a similar estimate due to symmetry. Next we look at the boundary layer term $w_{1}$. We obtain in $\Omega_{1}$ again by using (16)

$$
\begin{aligned}
\left|\left\langle\left(c_{K}-c\right)\left(w_{1}-\mathcal{I}_{1} w_{1}\right), \partial_{x} \boldsymbol{v}_{1}\right\rangle_{\Omega_{1}}\right| \\
\quad \lesssim \sum_{K \subset \Omega_{1}}\left(\left(h+N^{-1}\right)\left\|h_{x}^{k} \partial_{x}^{k+1} w_{1}\right\|_{L^{2}(K)}\right. \\
\left.\quad+\varepsilon^{-1} N^{-(k+1)}\left\|\partial_{y}^{k+1} w_{1}\right\|_{L^{2}\left(K \backslash \Omega_{c o r}\right)}+N h^{k+1}\left\|\partial_{y}^{k+1} w_{1}\right\|_{L^{2}\left(K \cap \Omega_{c o r}\right)}\right)\|\boldsymbol{v}\|_{L^{2}(K)} \\
\quad \lesssim\left(\left(h+N^{-1} \max \left|\psi^{\prime}\right|\right)^{k+1} \varepsilon^{-1}\left\|\mathrm{e}^{\frac{k x}{\sigma \varepsilon}} \mathrm{e}^{-\frac{x}{\varepsilon}}\right\|_{L^{2}\left(\Omega_{1}\right)}\right. \\
\left.\quad+\varepsilon^{-1} N^{-(k+1)}\left\|\mathrm{e}^{-\frac{x}{\varepsilon}}\right\|_{L^{2}\left(\Omega_{1} \backslash \Omega_{c o r}\right)}+N h^{k+1}\left\|\mathrm{e}^{-\frac{x}{\varepsilon}}\right\|_{L^{2}\left(\Omega_{1} \cap \Omega_{c o r}\right)}\right)\|\boldsymbol{v}\|_{L^{2}\left(\Omega_{1}\right)} \\
\quad \lesssim \varepsilon^{-1 / 2}\left(h+N^{-1} \max \left|\psi^{\prime}\right|\right)^{k+1}\left(1+h \varepsilon N^{2}\right)\|\boldsymbol{v}\|_{L^{2}\left(\Omega_{1}\right)} \\
\quad \lesssim \varepsilon^{-1 / 2}\left(h+N^{-1} \max \left|\psi^{\prime}\right|\right)^{k+1}\|\boldsymbol{v}\|_{L^{2}\left(\Omega_{1}\right)},
\end{aligned}
$$


using again the condition on $\varepsilon h$. For the $y$-derivative it holds similarly

$$
\begin{aligned}
& \left|\left\langle\left(c_{K}-c\right)\left(w_{1}-\mathcal{I}_{1} w_{1}\right), \partial_{y} \boldsymbol{v}_{2}\right\rangle_{\Omega_{1}}\right| \\
& \lesssim \sum_{K \subset \Omega_{1}}\left(\left(h+N^{-1}\right)\left\|h_{y}^{k} \partial_{y}^{k+1} w_{1}\right\|_{L^{2}(K)}+(1+h N)\left\|h_{x}^{k+1} \partial_{x}^{k+1} w_{1}\right\|_{L^{2}\left(K \backslash \Omega_{c o r}\right)}\right. \\
& \left.+\left(1+h \varepsilon^{-1} N\right)\left\|h_{x}^{k+1} \partial_{x}^{k+1} w_{1}\right\|_{L^{2}\left(K \cap \Omega_{c o r}\right)}\right)\|v\|_{L^{2}(K)} \\
& \lesssim\left(\left(h+N^{-1}\right)^{k+1}\left\|\mathrm{e}^{-\frac{x}{\varepsilon}}\right\|_{L^{2}\left(\Omega_{1}\right)}\right. \\
& +(1+h N)\left(N^{-1} \max \left|\psi^{\prime}\right|\right)^{k+1}\left\|\mathrm{e}^{\frac{(k+1) x}{\varepsilon}} \mathrm{e}^{-\frac{x}{\varepsilon}}\right\|_{L^{2}\left(\Omega_{1} \backslash \Omega_{c o r}\right)} \\
& \left.+\left(1+h \varepsilon^{-1} N\right)\left(N^{-1} \max \left|\psi^{\prime}\right|\right)^{k+1}\left\|\mathrm{e}^{\frac{(k+1) x}{\varepsilon}} \mathrm{e}^{-\frac{x}{\varepsilon}}\right\|_{L^{2}\left(\Omega_{1} \cap \Omega_{c o r}\right)}\right)\|\boldsymbol{v}\|_{L^{2}\left(\Omega_{1}\right)} \\
& \lesssim \varepsilon^{-1 / 2}\left(h+N^{-1} \max \left|\psi^{\prime}\right|\right)^{k+1}\left(\varepsilon+h \varepsilon N(\ln N)^{1 / 2}+h \varepsilon^{3 / 2} N \ln N\right)\|\boldsymbol{v}\|_{L^{2}\left(\Omega_{1}\right)} \\
& \lesssim \varepsilon^{-1 / 2}\left(h+N^{-1} \max \left|\psi^{\prime}\right|\right)^{k+1}\|\boldsymbol{v}\|_{L^{2}\left(\Omega_{1}\right)},
\end{aligned}
$$

where the condition on $\varepsilon h$ was used in the last step.

In the remainder of the domain we apply the $L^{2}$-stability and get by considering the different cases of $\frac{h_{y}}{h_{x}}$

$$
\begin{aligned}
\mid & \left\langle\left(c_{K}-c\right)\left(w_{1}-\mathcal{I}_{1} w_{1}\right), \partial_{x} \boldsymbol{v}_{1}\right\rangle_{\Omega \backslash \Omega_{1}} \mid \\
& \lesssim \sum_{K \subset \Omega \backslash \Omega_{1}}\left\|c_{K}-c\right\|_{L^{\infty}(K)}\left\|w_{1}-\mathcal{I}_{1} w_{1}\right\|_{L^{2}(K)}\left\|h_{x}^{-1} \boldsymbol{v}_{1}\right\|_{L^{2}(K)} \\
& \lesssim \sum_{K \subset \Omega \backslash \Omega_{1}}\left(1+\frac{h_{y}}{h_{x}}\right)\left\|w_{1}\right\|_{L^{2}(K)}\|\boldsymbol{v}\|_{L^{2}(K)} \\
& \lesssim\left(1+h N+\varepsilon^{-1}+h \varepsilon^{-1} N\right)\left\|w_{1}\right\|_{L^{2}\left(\Omega \backslash \Omega_{1}\right)}\|\boldsymbol{v}\|_{L^{2}\left(\Omega \backslash \Omega_{1}\right)} \\
& \lesssim \varepsilon^{-1 / 2} N^{-(k+1)}\|\boldsymbol{v}\|_{L^{2}\left(\Omega \backslash \Omega_{1}\right)} \\
\mid & \left\langle\left(c_{K}-c\right)\left(w_{1}-\mathcal{I}_{1} w_{1}\right), \partial_{y} \boldsymbol{v}_{2}\right\rangle_{\Omega \backslash \Omega_{1}} \mid \\
& \lesssim \sum_{K \subset \Omega \backslash \Omega_{1}}\left(1+\frac{h_{x}}{h_{y}}\right)\left\|w_{1}\right\|_{L^{2}(K)}\|\boldsymbol{v}\|_{L^{2}(K)} \\
& \lesssim \varepsilon^{-1 / 2} N^{-(k+1)}\|\boldsymbol{v}\|_{L^{2}\left(\Omega \backslash \Omega_{1}\right)} .
\end{aligned}
$$

The estimation of the other boundary layer terms and of the corner layer terms is similar. Combining all the individual results proves the assertion.

Lemma 6 For $h \varepsilon \lesssim N^{-2}$ it holds for $\mathcal{D}(K)=R T_{k}(K)$ with $\sigma \geq k+3 / 2$

$$
\||(\xi, \xi)|\|_{b a l} \lesssim\left(h+N^{-1} \max \left|\psi^{\prime}\right|\right)^{k+1}(\ln N)^{1 / 2}
$$

and for $\mathcal{D}(K)=B D M_{k}(K)$ with $\sigma \geq k+1$

$$
\||(\xi, \xi)|\|_{b a l} \lesssim\left(h+N^{-1} \max \left|\psi^{\prime}\right|\right)^{k} .
$$

Proof Using the inf-sup estimate (14) and the previous lemmas we obtain for $\mathcal{D}(K)=$ $R T_{k}(K)$

$$
\beta\||(\xi, \xi)|\| \leq \sup _{V \in \mathcal{U}_{N}} \frac{B((\eta, \eta), V)}{\|V\|_{L^{2}(\Omega)}} \lesssim \varepsilon^{1 / 2}\left(h+N^{-1} \max \left|\psi^{\prime}\right|\right)^{k+1}(\ln N)^{1 / 2},
$$


particularly

$$
\begin{aligned}
& \|\xi\|_{L^{2}(\Omega)} \lesssim \varepsilon^{1 / 2}\left(h+N^{-1} \max \left|\psi^{\prime}\right|\right)^{k+1}(\ln N)^{1 / 2}, \\
& \varepsilon^{-1 / 2}\|\xi\|_{L^{2}(\Omega)} \lesssim\left(h+N^{-1} \max \left|\psi^{\prime}\right|\right)^{k+1}(\ln N)^{1 / 2}, \\
& \delta^{1 / 2} \varepsilon^{-1 / 2}\|\varepsilon \operatorname{div} \xi\|_{L^{2}(\Omega)} \lesssim\left(h+N^{-1} \max \left|\psi^{\prime}\right|\right)^{k+1}(\ln N)^{1 / 2},
\end{aligned}
$$

which are the three components of $\|\mid \xi\|_{\text {bal }}$. Similar results follow for $\mathcal{D}(K)=B D M_{k}(K)$ with the additional

$$
\left(h+N^{-1} \max \left|\psi^{\prime}\right|\right)(\ln N)^{1 / 2} \lesssim 1
$$

Theorem 7 For $h \varepsilon \lesssim N^{-2}$ it holds for $\mathcal{D}(K)=R T_{k}(K)$ with $\sigma \geq k+3 / 2$

$$
\left\|\left|U-U_{N}\right|\right\|_{b a l} \lesssim\left(h+N^{-1} \max \left|\psi^{\prime}\right|\right)^{k+1}(\ln N)^{1 / 2} .
$$

and for $\mathcal{D}(K)=B D M_{k}(K)$ with $\sigma \geq k+1$

$$
||\left|U-U_{N}\right| \|_{b a l} \lesssim\left(h+N^{-1} \max \left|\psi^{\prime}\right|\right)^{k} .
$$

Proof With the triangle inequality

$$
\left\|\left|U-U_{N}\left\|_{b a l} \leq\right\|\right|\left|(\xi, \xi)\left\|_{b a l}+\right\|\right|(\eta, \eta)\right\|_{b a l}
$$

and the previous lemmas it only remains to estimate $\|\eta\|_{L^{2}(\Omega)}$, which can be done using the local anisotropic interpolation error estimates (7) by standard techniques

$$
\|\eta\|_{L^{2}(\Omega)} \lesssim\left(h+N^{-1} \max \left|\psi^{\prime}\right|\right)^{k+1} .
$$

Corollary 8 Under the same conditions as the previous theorem we also have for $\mathcal{D}(K)=$ $R T_{k}(K)$ in the unbalanced norm

$$
\left\|\left|U-U_{N}\right|\right\| \lesssim\left(h+N^{-1} \max \left|\psi^{\prime}\right|\right)^{k+1} .
$$

Remark 9 On a Shishkin mesh we have $h=h_{i} \lesssim \varepsilon N^{-1} \ln N$ inside the boundary domain. Thus the condition on $\varepsilon h$ becomes

$$
\varepsilon^{2} \lesssim \frac{N^{-1}}{\ln N} \text { and } h+N^{-1} \max \left|\psi^{\prime}\right| \lesssim N^{-1} \ln N .
$$

On a Bakhvalov S-mesh we have $h \sim \varepsilon$ and the condition becomes

$$
\varepsilon \lesssim N^{-1} \text { and therefore } h+N^{-1} \max \left|\psi^{\prime}\right| \lesssim N^{-1} \text {. }
$$

Note also, that $\varepsilon h \lesssim N^{-2}$ and $h \lesssim \varepsilon$ always imply $h \lesssim N^{-1}$.

Remark 10 The same analysis can also be conducted for the Arnold-Boffi-Falk element

$$
\mathcal{D}(K)=A B F_{k}(K):=\mathcal{Q}_{k+2, k}(K) \times \mathcal{Q}_{k, k+2}(K),
$$

see [3], using $\operatorname{div} \mathcal{D}(K)=\mathcal{Q}_{k+1}(K) \backslash \operatorname{span}\left\{x^{k+1} y^{k+1}\right\}$ as discrete space for the first component. Anisotropic interpolation error estimates are given in [7]. Although $\|\eta\|_{L^{2}(\Omega)}$ and $\|\operatorname{div} \eta\|_{L^{2}(\Omega)}$ can be estimated with order $k+2$, we obtain only convergence rates of order $k+1$ due to $\|\boldsymbol{\eta}\|_{L^{2}(\Omega)} \lesssim \varepsilon^{1 / 2}\left(h+N^{-1} \max \left|\psi^{\prime}\right|\right)^{k+1}$. 
Table 1 Errors in various norms for varying values of $\varepsilon$ and fixed $N, R T_{1}$

\begin{tabular}{llllll}
\hline$\varepsilon$ & $\left\|u-u_{h}\right\|_{L^{2}(\Omega)}$ & $\left\|\boldsymbol{u}-\boldsymbol{u}_{h}\right\|_{L^{2}(\Omega)}$ & $\left\|\operatorname{div}\left(\boldsymbol{u}-\boldsymbol{u}_{h}\right)\right\|_{L^{2}(\Omega)}$ & \|\|$U-U_{h} \| \mid$ & $\left\|U-U_{h}\right\|_{\text {bal }}$ \\
\hline $10^{-3}$ & $5.904 \mathrm{e}-04$ & $4.492 \mathrm{e}-05$ & $1.445 \mathrm{e}-01$ & $5.946 \mathrm{e}-04$ & $2.311 \mathrm{e}-03$ \\
$10^{-4}$ & $5.867 \mathrm{e}-04$ & $1.401 \mathrm{e}-05$ & $4.591 \mathrm{e}-01$ & $5.871 \mathrm{e}-04$ & $2.304 \mathrm{e}-03$ \\
$10^{-5}$ & $5.863 \mathrm{e}-04$ & $4.421 \mathrm{e}-06$ & $1.452 \mathrm{e}-00$ & $5.863 \mathrm{e}-04$ & $2.303 \mathrm{e}-03$ \\
$10^{-6}$ & $5.863 \mathrm{e}-04$ & $1.398 \mathrm{e}-06$ & $4.593 \mathrm{e}-00$ & $5.863 \mathrm{e}-04$ & $2.303 \mathrm{e}-03$ \\
\hline
\end{tabular}

\section{Numerical Experiments}

Let us consider on $\Omega=(0,1)^{2}$

$$
-\varepsilon^{2} \Delta u+c u=f,
$$

where

$$
c=1+x^{2} y^{2} \mathrm{e}^{x y / 2} \Rightarrow c_{0}=1, c_{\infty}=1+\mathrm{e}^{1 / 2}, \delta=\frac{1}{\left(1+\mathrm{e}^{1 / 2}\right)^{2}}
$$

and an exact solution

$$
u=\left(\cos \left(\frac{\pi x}{2}\right)-\frac{\mathrm{e}^{-x / \varepsilon}-\mathrm{e}^{-1 / \varepsilon}}{1-\mathrm{e}^{-1 / \varepsilon}}\right) \cdot\left(1-y-\frac{\mathrm{e}^{-y / \varepsilon}-\mathrm{e}^{-1 / \varepsilon}}{1-\mathrm{e}^{-1 / \varepsilon}}\right)
$$

is prescribed, see [1,17] for $c=1$. The solution has only boundary layers at $x=0$ and $y=0$, and a corner layer at $(0,0)$. Therefore, we modify our mesh accordingly. For our experiments we will always use Bakhvalov-S-meshes.

All computations were done in $\mathbb{S O F} \mathbb{E}$, a finite-element framework in Matlab and Octave, see gi thub. com/SOFE-Developers/SOFE.

Let us start the numerical investigation by looking at the dependence on $\varepsilon$. For that we fix $N=16$ and use $R T_{1}$-elements, and vary $\varepsilon \in\left\{10^{-3}, 10^{-4}, 10^{-5}, 10^{-6}\right\}$. We obtain the numbers in Table 1.

As expected from Theorem 7, we observe independence of $\varepsilon$ in $\left\|u-u_{h}\right\|_{L^{2}(\Omega)}$, and a dependence on $\varepsilon$ in $\left\|\boldsymbol{u}-\boldsymbol{u}_{h}\right\|_{L^{2}(\Omega)} \lesssim \varepsilon^{1 / 2}$ and $\left\|\operatorname{div}\left(\boldsymbol{u}-\boldsymbol{u}_{h}\right)\right\|_{L^{2}(\Omega)} \lesssim \varepsilon^{-1 / 2}$. Consequently, $\|\left|U-U_{h}\right|||$ stays independent of $\varepsilon$, due to the dominating effect of $\left\|u-u_{h}\right\|_{L^{2}(\Omega)}$, and the larger balanced norm $\left\|U-U_{h}\right\|_{b a l}$ is independent too due to the correct weighting of the other two norms.

Now let us come to the convergence orders. For that purpose we fix $\varepsilon=10^{-4}$ and vary for different values of $k$ the number $N$ of cells per dimension. We start with Raviart-Thomas elements and obtain the results of Table 2.

Here along with the computed errors also the estimated rates of convergence are given and they are close to the expected rates of $k+1$ for the balanced norm.

In the case of Brezzi-Douglas-Marini elements we get Table 3.

As expected we only see rates of $k$ in the balanced version with slightly better results for the lowest order case. The reason for this behaviour lies in the components of the balanced norms, where the faster converging ones dominate for smaller values of $N$ the balanced norm. A closer look reveals $\left\|u-u_{h}\right\|_{L^{2}(\Omega)}$ and $\left\|\operatorname{div}\left(\boldsymbol{u}-\boldsymbol{u}_{h}\right)\right\|_{L^{2}(\Omega)}$ only to be convergent with order 1 , see Table 4. 
Table 2 Errors $\left\|\left|U-U_{h}\right|\right\|_{b a l}$ for fixed $\varepsilon=10^{-4}$ in the Raviart-Thomas case

\begin{tabular}{|c|c|c|c|c|c|c|}
\hline$N$ & $k=1$ & & $k=2$ & & $k=3$ & \\
\hline 8 & $9.250 \mathrm{e}-03$ & & $1.059 \mathrm{e}-03$ & & $1.640 \mathrm{e}-04$ & \\
\hline 16 & $2.304 \mathrm{e}-03$ & 2.01 & $1.518 \mathrm{e}-04$ & 2.80 & $1.238 \mathrm{e}-05$ & 3.73 \\
\hline 32 & $5.679 \mathrm{e}-04$ & 2.02 & $2.023 \mathrm{e}-05$ & 2.91 & $8.433 \mathrm{e}-07$ & 3.88 \\
\hline 64 & $1.402 \mathrm{e}-04$ & 2.02 & $2.603 e-06$ & 2.96 & $5.480 \mathrm{e}-08$ & 3.94 \\
\hline 128 & $3.479 \mathrm{e}-05$ & 2.01 & $3.298 \mathrm{e}-07$ & 2.98 & $3.488 \mathrm{e}-09$ & 3.97 \\
\hline 256 & $8.657 \mathrm{e}-06$ & 2.01 & $4.148 \mathrm{e}-08$ & 2.99 & $2.198 \mathrm{e}-10$ & 3.99 \\
\hline 512 & $2.158 \mathrm{e}-06$ & 2.00 & & & & \\
\hline
\end{tabular}

Table 3 Errors ||$\left|U-U_{h}\right| \|_{b a l}$ for fixed $\varepsilon=10^{-4}$ in the Brezzi-Douglas-Marini case

\begin{tabular}{|c|c|c|c|c|c|c|}
\hline$N$ & $k=1$ & & $k=2$ & & $k=3$ & \\
\hline 8 & $8.406 \mathrm{e}-01$ & & $1.520 \mathrm{e}-02$ & & $2.700 \mathrm{e}-03$ & \\
\hline 16 & $6.451 \mathrm{e}-01$ & 0.38 & $3.420 \mathrm{e}-03$ & 2.15 & $3.624 \mathrm{e}-04$ & 2.90 \\
\hline 32 & $3.519 \mathrm{e}-01$ & 0.87 & $8.492 \mathrm{e}-04$ & 2.01 & $4.606 \mathrm{e}-05$ & 2.98 \\
\hline 64 & $1.796 \mathrm{e}-01$ & 0.97 & $2.144 \mathrm{e}-04$ & 1.99 & $5.553 e-06$ & 3.05 \\
\hline 128 & $7.124 \mathrm{e}-02$ & 1.33 & $5.403 e-05$ & 1.99 & $6.631 \mathrm{e}-07$ & 3.07 \\
\hline 256 & $2.186 \mathrm{e}-02$ & 1.70 & $1.356 \mathrm{e}-05$ & 1.99 & $8.129 \mathrm{e}-08$ & 3.03 \\
\hline 512 & $5.906 \mathrm{e}-03$ & 1.89 & $3.390 \mathrm{e}-06$ & 2.00 & $1.027 \mathrm{e}-08$ & 2.98 \\
\hline 1024 & $1.566 \mathrm{e}-03$ & 1.92 & & & & \\
\hline
\end{tabular}

Table 4 Errors is various norms for fixed $\varepsilon=10^{-4}$ in the $B D M_{1}$-case

\begin{tabular}{lllllll}
\hline$N$ & $\left\|u-u_{h}\right\|_{L^{2}(\Omega)}$ & \multicolumn{2}{c}{$\varepsilon^{-1 / 2}\left\|\boldsymbol{u}-\boldsymbol{u}_{h}\right\|_{L^{2}(\Omega)}$} & $(\delta \varepsilon)^{1 / 2}\left\|\operatorname{div}\left(\boldsymbol{u}-\boldsymbol{u}_{h}\right)\right\|_{L^{2}(\Omega)}$ \\
\hline 8 & $2.786 \mathrm{e}-03$ & & $8.386 \mathrm{e}-01$ & & $5.716 \mathrm{e}-02$ & \\
16 & $9.940 \mathrm{e}-04$ & 1.49 & $6.444 \mathrm{e}-01$ & 0.38 & $3.034 \mathrm{e}-02$ & 0.91 \\
32 & $4.369 \mathrm{e}-04$ & 1.19 & $3.516 \mathrm{e}-01$ & 0.87 & $1.554 \mathrm{e}-02$ & 0.96 \\
64 & $2.114 \mathrm{e}-04$ & 1.05 & $1.794 \mathrm{e}-01$ & 0.97 & $7.860 \mathrm{e}-03$ & 0.98 \\
128 & $1.051 \mathrm{e}-04$ & 1.01 & $7.113 \mathrm{e}-02$ & 1.33 & $3.953 \mathrm{e}-03$ & 0.99 \\
256 & $5.254 \mathrm{e}-05$ & 1.00 & $2.177 \mathrm{e}-02$ & 1.71 & $1.982 \mathrm{e}-03$ & 1.00 \\
512 & $2.629 \mathrm{e}-05$ & 1.00 & $5.822 \mathrm{e}-03$ & 1.90 & $9.926 \mathrm{e}-04$ & 1.00 \\
1024 & $1.315 \mathrm{e}-05$ & 1.00 & $1.485 \mathrm{e}-03$ & 1.97 & $4.965 \mathrm{e}-04$ & 1.00 \\
\hline
\end{tabular}

Funding Open Access funding enabled and organized by Projekt DEAL. Partial funding by DAAD PPP Serbia 2021-23.

Data Availability The datasets generated during and analysed during the current study are available from the corresponding author on reasonable request.

\section{Declarations}

Conflict of interest The authors declare that they have no conflict of interest. 
Code Availability The software is available at github.com/SOFE-Developers/SOFE.

Open Access This article is licensed under a Creative Commons Attribution 4.0 International License, which permits use, sharing, adaptation, distribution and reproduction in any medium or format, as long as you give appropriate credit to the original author(s) and the source, provide a link to the Creative Commons licence, and indicate if changes were made. The images or other third party material in this article are included in the article's Creative Commons licence, unless indicated otherwise in a credit line to the material. If material is not included in the article's Creative Commons licence and your intended use is not permitted by statutory regulation or exceeds the permitted use, you will need to obtain permission directly from the copyright holder. To view a copy of this licence, visit http://creativecommons.org/licenses/by/4.0/.

\section{References}

1. Adler, J., MacLachlan, S., Madden, N.: First-order system least squares finite-elements for singularly perturbed reaction-diffusion equations. In: Lirkov, I., Margenov, S. (eds.) Large-Scale Scientific Computing, Springer International Publishing, pp. 3-14 (2020)

2. Apel, T.: Anisotropic finite elements: local estimates and applications. In: Advances in Numerical Mathematics, B. G. Teubner, Stuttgart (1999)

3. Arnold, D.N., Boffi, D., Falk, R.S.: Quadrilateral $H_{d i v}$ finite elements. SIAM J. Numer. Anal. 42(6), 2429-2451 (2005)

4. Boffi, D., Brezzi, F., Fortin, M.: Mixed Finite Element Methods and Applications, Springer Series in Computational Mathematics, vol. 41. Springer, Berlin (2013)

5. Brezzi, F., Douglas, J., Marini, L.D.: Two families of mixed finite elements for second order elliptic problems. Numerische Mathematik 47, 217-235 (1985)

6. Clavero, C., Gracia, J.L., ORiordan, E.: A parameter robust numerical method for a two dimensional reaction-diffusion problem. Math. Comput. 74(252), 1743-1758 (2005)

7. Franz, S.: Anisotropic $H_{d i v}$-norm error estimates for rectangular $H_{d i v}$-elements. Appl. Math. Lett. 121, 107453 (2021). https://doi.org/10.1016/j.aml.2021.107453

8. Franz, S., Matthies, G.: Convergence on layer-adapted meshes and anisotropic interpolation error estimates of non-standard higher order finite elements. Appl. Numer. Math. 61, 723-737 (2011)

9. Franz, S., Roos, H.G.: Error estimates in balanced norms of finite element methods for higher order reaction-diffusion problems. Int. J. Numer. Anal. Model. 17, 532-542 (2020)

10. Han, H., Kellogg, R.B.: Differentiability properties of solutions of the equation $-\varepsilon^{2} \Delta u+r u=f(x, y)$ in a square. SIAM J. Math. Anal. 21(2), 394-408 (1990)

11. Lin, R., Stynes, M.: A balanced finite element method for singularly perturbed reaction-diffusion problems. SIAM J. Numer. Anal. 50(5), 2729-2743 (2012)

12. Linß, T.: Layer-adapted meshes for reaction-convection-diffusion problems. In: Lecture Notes in Mathematics, vol. 1985, Springer, Heidelberg (2010)

13. Liu, F., Madden, N., Stynes, M., Zhou, A.: A two-scale sparse grid method for a singularly perturbed reaction-diffusion problem in two dimensions. IMA J. Numer. Anal. 29(4), 986-1007 (2008)

14. Raviart, P.A., Thomas, J.M.: Primal hybrid finite element methods for 2 nd order elliptic equations. Math. Comp. 31(138), 391-413 (1977)

15. Roos, H.G.: Error estimates in balanced norms of finite element methods on layer-adapted meshes for second order reaction-diffusion problems. In: Huang, Z., Stynes, M., Zhang, Z. (eds.) Boundary and Interior Layers, Computational and Asymptotic Methods, BAIL 2016, Springer International Publishing, pp. 1-18 (2017)

16. Roos, H.G., Linß, T.: Sufficient conditions for uniform convergence on layer-adapted grids. Computing 63, 27-45 (1999)

17. Roos, H.G., Schopf, M.: Convergence and stability in balanced norms of finite element methods on Shishkin meshes for reaction-diffusion problems. ZAMM 95(6), 551-565 (2015)

18. Roos, H.G., Stynes, M., Tobiska, L.: Robust numerical methods for singularly perturbed differential equations. In: Springer Series in Computational Mathematics, 2nd edn., vol. 24, Springer, Berlin (2008)

19. Stynes, M.: Sharp anisotropic interpolation error estimates for rectangular Raviart-Thomas elements. Math. Comp. 290(83), 2675-2689 (2014)

Publisher's Note Springer Nature remains neutral with regard to jurisdictional claims in published maps and institutional affiliations. 